\title{
Conceptual Replication of Canine Serum Oxytocin Increase Following a Positive Dog-human Interaction
}

\author{
LUMINITA DIANA HRITCU1, CRISTINA HORHOGEA ${ }^{1 *}$, ALIN CIOBICA ${ }^{2,3,4}$, MIHAELA CLAUDIA SPATARU1, CONSTATIN SPATARU ${ }^{1}$, \\ ANNA KIS \\ ${ }^{1}$ Ion Ionescu de la Brad University of Agricultural Science and Veterinary Medicine, 3 Sadoveanu Alley, 700490, Iasi, Romania \\ ${ }^{2}$ Alexandru Ioan Cuza University of lasi, Faculty of Biology, Department of Research, 11 Carol I Blvd., 700506, Iasi, Romania \\ ${ }^{3}$ Academy of Romanian Scientists, 54 Splaiul Independentei St., 050094, Bucuresti, Romania \\ ${ }^{4}$ Romanian Academy, Iasi, Center of Biomedical Research, 8 Carol I Blvd., 700506, lasi, Romania \\ ${ }^{5}$ Hungarian Academy of Sciences, Institute of Cognitive Neuroscience and Psychology, 2.Magyar Tudosok korutja St., 1117 \\ Budapest, Hungary
}

\begin{abstract}
The neurohormone oxytocin is known to exert a special function in the regulation of social relationships throughout vertebrate taxa. Recently it has been discovered that not only within-species, but in certain cases, between-species social bonds are also mediated by the same hormone, e.g. in case of the doghuman relationship. However, despite the exponential grow th of findings at the behavioural level, there are still a lot of controversies on the biochemical assessment of oxyto in in canine samples. Thus, in the current study we replicate previous findings of canine serum oxytocin increase following a positive dog-human interaction. We provide a detailed description of both the immunoassay method used as well as the behavioural protocol (including crucial time-parameters). This will serve as a base for further studies that both our group as well as others in the field will conduct.
\end{abstract}

Keywords: oxytocin, neurohormone, social behaviour, dog.

The neurohormone oxytocin has been proposed to be a key regulator of the special social bond between dogs and humans [1]. Such claims are in big part based on data showing that following different forms of positive social interactions peripheral (blood or urine) oxytocin levels are elevated in the dogs [2-4]. While the field has since proven the interrelatedness of the oxytocin system and dogs' human-like social behaviour at various levels [5], the measurement of peripheral oxytocin in dogs, as well as its relation to pre-measurement social stimuli still remains a controversial issue. Looking beyond the generalised findings, raw data on dogs' baseline blood oxytocin levels show a huge between-study variation (differences of up to four magnitudes have be observed; e.g. $0.1 \mathrm{ng} / \mathrm{L}$ in Odendaal and Meintjes, 2003 versus $155.8 \mathrm{pmol} / \mathrm{L}$ in Handlin et al. [2], 2011 respectively). In parallel to this the commercially available immunoassay methods have been questioned in terms of reliability. Furthermore recently a study focusing on null results about the effect of positive interaction on urinary oxytocin levels has also been published [6], while previously such results even if published were not discussed as main results [7].

The importance of replication studies is increasing as the so called file-drawer bias and publication bias has been a serious issue in the behavioural sciences in general. This means that many authors struggle or not even attempt to publish negative results or those contradicting the field [8, 9]. Similarly, reports about null effects of social interaction on dog oxytocin levels might be difficult to get published (see e.g. Marshall etal., 2018 for an unpublished conference abstract [10]). It is unlikely that the effect of positive social interaction on dog's oxytocin level would be untrue, many studies have shown that such a result exists. How ever, differences between studies that might appear minor at first, including differences in the oxytocin measurement per se are probably more relevant than the field has initially considered. To start with peripheral oxytocin measurements are often pulled together as such, even though it has been recently shown that serum concentration and urine concentration do no necessarily react in the same way [11]. This together with the fact that the studies apply different waiting times between treatment (interaction) and measurement might in it self be enough to cause differences in results. Furthermore the interaction protocols of these studies, as well as the relationship between the dog and the handler (e.g. owner versus research assistant) vary greatly. Thus there is ample room for future studies to examine the nuances of the oxytocin inducing effect of dog-human social interactions. As a first step for such a grand-scale project, in this paper we attempt a conceptual replication of the general finding that dogs' serum oxytocin level increases following positive social interaction with the owner involving both eyecontact, stroking and dog-directed talk.

\section{Exprimental part \\ Ethical permission}

This study was approved by the local ethical committee under the registration number 882/12.09.2018.

\section{Subjects}

Our subjects were $\mathrm{N}=6$ privately owned pet dogs ( 3 males, 3 females), all older than 1 year of age (mean \pm sd $=6.5 \pm 3.9$ years) .

\section{Oxytocin measurement}

Serum samples were obtained by venous blood harvested in red topped tubes; the samples were allowed to clot for $2 \mathrm{~h}$ at room temperature before centrifugation for $20 \mathrm{~min}$ at $1000 \times \mathrm{g}$ (3000 rpm). The supernatant was collected and stored at $-20^{\circ} \mathrm{C}$ before testing.

The oxytocin level was evaluated using a competitive enzyme immunoassay (EIA): Oxytocin ELISA Kit (LSBio LifeSpan Bio Sciences, Inc.) following the manufacturer instructions.

\footnotetext{
*email; rebegeacristina@yahoo.com, Phone: 0040745544841
} 
The 96-wells plate has been pre-coated with a target specific antigen. Before begining the assay, some lyophilized components of the kit must be reconstituted and some others must be diluted in order to obtain work solutions.

\section{Standard preparation}

In order to determine the concentration of oxytocin in the samples, we used eight standard solutions with well know oxytocin concentrations to generate a standard curve. The standard stock solution (SSS) $(1000 \mathrm{ig} / \mathrm{mL})$ was obtain dissolving standard lyophilized solution in $1 \mathrm{~mL}$ of diluent. Following, the stock solution was incubated at room temperature for $10 \mathrm{~min}$ with gentle shaking, avoiding foaming. The rest of the standard dilutions (D) were prepared in accordance with table 1.

\section{Reagent Preparation}

The biotinylated detection antibody and the avidin horseradish peroxidase conjugate (A-HRP-C) were diluted using specific diluents in order to obtain the working solution 1:100. Also, the concentrate wash buffer (25x) was diluted with distilled water.

\section{Assay Procedure}

a. $50 \mu \mathrm{L}$ of standard dilutions (D1-D7), zero standard and samples were added in wells.

b. $50 \mu \mathrm{L}$ of biotinylated detection antibody were added to each well, the plate was covered with a plate sealer, gently mixed and incubated for $45 \mathrm{~min}$ at $37^{\circ} \mathrm{C}$ (microplate incubator and shaker Stat Fax 2200 Awareness Technology Inc.). The free oxytocin (in the standards or samples) and antigens bound to the plate compete to bind to the detection antibody.

c. The liquid was aspirated fand each well was washed three times with $350 \mu \mathrm{L}$ of wash buffer using an automated washer (Stat Fax 2600 Awareness Technology Inc.). All but the bound biotinylated detection antibody was washed away.

d.100u L of A-HRP-C working solution was added to each well and the plate were incubated for $30 \mathrm{~min}$ at $37^{\circ} \mathrm{C}$. This conjugate bound to the biotin complex.

e. The liquid was aspirated and each well and washed three times as outlined in step c. Unbound A-HRP-C is washed away.

f. $90 \mu \mathrm{L}$ of 3,3,5,5'-Tetramethylbenzidine (TMB) substrate was added to each well and the plate was incubated for 15 minutes at $37^{\circ} \mathrm{C}$, protected from light. If the previous reagent interacted, the HRP enzyme will react with the substrate and a blue color develop.

g. $50 \mu \mathrm{L}$ of stop solution (sulphuric acid) were added to each well and the blue color changed to yellow immediately.

$h$. The optical density (OD value) of each well was determined immediately using a microplate reader (Stat Fax 3200 Awareness Technology Inc.) set to $450 \mathrm{~nm}$.
The OD of the serum sample were compared to the OD standard curve generated. In competition assay the greater the amount of antigen in the sample, the lower the color development and optical density reading. The conversion of the OD values in $\mathrm{pg} / \mathrm{mL}$ was accomplished using a formula according to the Excel.

\section{Social interaction}

Following blood sample collection for the baseline oxytocin measure, subjects participated in a 10min long positive social interaction with their owner. The owner was instructed to talk to the dog as she/he naturally would (using dog-directed speech), petting the dog and looking into its eyes as much as possible.

\section{Results and discussions}

Dogs' serum oxytocin concentration was significantly higher after the interaction compared to baseline (paired samples t-test; $t_{(5)}=4.846, p=0.005$ ) (fig. 1).

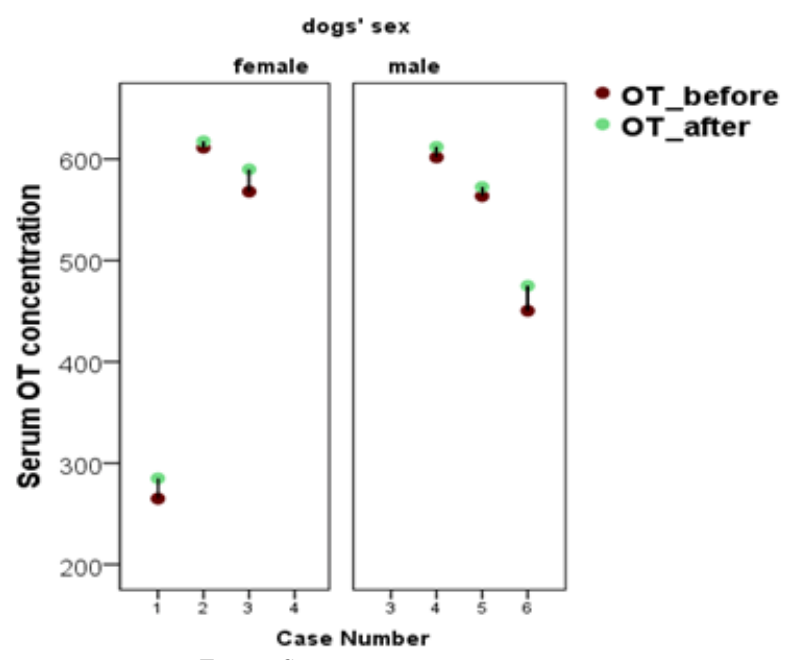

Fig. 1. Serum oxytocin concentration

Thus, the present study has carried out a conceptual replication of previous findings showing an elevated peripheral oxytocin level in the dog following a positive dog-human social interaction. While such early results have been for quite some time the basis of big claims about the dog-human bond, recent failures to replicate those findings along with the arising methodological concerns about peripheral oxytocin measurements in general make it necessary to carry out replication studies.

Our study used a small sample size, as early reports of positive social stimuli on peripheral oxytocin increase have reported a strong result on similarly low samples suggesting a big effect size (e.g. $\mathrm{N}=5$ male and $\mathrm{N}=4$ female dogs were tested in Mitsui etal., (2011) [3]. Present results seem to confirm these findings, as despite considerable individual variation, a consistent and statistically significant increase in blood-oxytocin levels was observed in $\mathrm{N}=6 \mathrm{dogs}$. The

\begin{tabular}{|c|c|c|}
\hline Standard dilution & $\begin{array}{l}\text { Concentration } \\
(\mathrm{pg} / \mathrm{mL})\end{array}$ & Method \\
\hline $\mathrm{D} 1$ & $1000^{-}$ & $500 \mu \mathrm{L}$ of $\mathrm{SSS}+0 \mu \mathrm{L}$ of sample diluent \\
\hline $\mathrm{D} 2$ & 500 & $250 \mu \mathrm{L}$ of $\mathrm{D} 1+250 \mu \mathrm{L}$ of sample diluent \\
\hline $\mathrm{D} 3$ & $250^{-}$ & $250 \mu \mathrm{L}$ of $2+250 \mu \mathrm{L}$ of sample diluent \\
\hline $\mathrm{D}^{-}$ & $-125^{-}$ & $250 \mu \mathrm{L}$ of $\mathrm{D} 3+250 \mu \mathrm{L}$ of sample diluent \\
\hline D5 ${ }^{-}$ & 62.5 & $250 \mu \mathrm{L}$ of $\mathrm{D} 4+250 \mu \mathrm{L}$ of sample diluent \\
\hline D6 ${ }^{-}$ & $31.25^{-}$ & $250 \mu \mathrm{L}$ of $\mathrm{D} 5+250 \mu \mathrm{L}$ of sample diluent \\
\hline $\mathrm{D} 7^{-}$ & $15.63^{-}$ & $250 \mu \mathrm{L}$ of $\mathrm{D} 6+250 \mu \mathrm{L}$ of sample diluent \\
\hline
\end{tabular}

Table 1

STANDARD DILUTIONS PREPARATION 
experiment by Romero et al. (2014) [8], where no effect of social interaction was found on oxytocin levels, used a 30-minute-long interaction, that is considerably longer than those used in both previous studies and our present research. It is plausible to assume that the length of the interaction might be an important factor, as petting of the dog for such a prolonged period might be an unnatural situation for both the subject and the handler. Powell et al. (2019) [7] propose that their null results in terms of oxytocin level increase might be due to studying urine instead of blood samples, and indeed their timing of sample collection (approximately $30 \mathrm{~min}$ after interaction) might have been too early $[3,11]$. This also suggests that while collecting urine or saliva samples for hormonal analysis might be preferable for the non-invasive nature of these methods, blood samples are generally more reliable.

Several studies suggest that the oxytocin system has a sex-specific effect, and some have even found that the increase of peripheral oxytocin after a positive interaction is specific to female dogs [12]. Our subjects were both males and females and no apparent difference could be observed between the two sexes. It is also important to note that male and female humans are known to interact differently with dogs, and dogs, in turn, differentiate between the two sexes [13-15]. All our subjects participated with a male owner, thus we did not induce variation to our sample in this respect.

\section{Conclusions}

The present results have a two-fold message for future research. First, the social interaction protocol used in our study have proven to be successful in inducing oxytocin increase, and can thus be used in future studies to explore the effect of such interactions in different dog populations and/or using post-interaction behavioural measures. Second, our immunoassay measurement (with the used kit, and applying the used timing) proved to be reliable for detecting serum oxytocin concentrations in the dog, and thus can be used in future studies for tracking individual oxytocin level changes.

The authors are stating that there are no conflict of interests to disclose except for Alin CIOBICA which is supported by a Young Teams grant offered by UEFISCDI Romania, no. PN-III-P1-1.1-TE-2016-1210, contract no. 58 din 02/05/2018 and Anna KIS being part of a Bial grant no. 169/ 16.

\section{References}

1. BEETZ, A., JULIUS, H., TURNER, D., KOTRSCHAL, K., Frontiers in Psychology, 3, 2012, Article Number: 352, DOI: 10.3389/fpsyg.2012.00352. 2. HANDLIN, L., HYDBRING-SANDBERG, E., NILSSON, A., EJ DEBACK, M., JANSSON, A., UVNAS-MOBERG, K., Anthrozoos, 24, No. 3, 2011, p. 301, DOI: $10.2752 / 175303711 X 13045914865385$.

3. MITSUI, S., YAMAMOTO, M., NAGASAWA, M., MOGI, K., KIKUSUI, T., OHTANI, N., OHTA, M., Hormones and Behavior, 60, No. 3, 2011, p. 239, DOI: 10.1016/j.yhbeh.2011.05.012.

4. ODENDAAL, J.S., MEINTJES, R.A., Veterinary J ournal,. 165, No. 3, 2003, p. 296.

5. KIS, A., CIOBICA, A., TOPAL, J., Hormones and Behavior, 94, 2017, p. 40.

6. MCCULLOUGH, M.E., CHURCHLAND, P.S., MENDEZ, A.J ., Neurosci. Biobehav. Rev., 37, No. 8, 2013. p. 1485.

7. POWELL, L., EDWARDS, K.M., BAUMAN, A., GUASTELLA, A.J ., DRAYTON, B., STAMATAKIS, E., MCGREEVY, P., Animals, 9, No. 2, 2019, Article Number: 51, DOI: 10.3390/ani9020051.

8. ROMERO, T., NAGASAWA, M., MOGI, K., HASEGAWA, T., KIKUSUI, T., Proceedings of the National Academy of Sciences of the United States of America, 111, No. 25, 2014, p. 9085, DOI: 10.1073/pnas.1322868111. 9. EASTERBROOK, P.J., BERLIN, J.A., GOPALAN, R., MATTHEWS, D.R., Lancet, 337, No. 8746, 1991, p. 867, DOI: 10.1016/0140-6736(91)90201-Y. 10. MARSHALL-PESCINI, S., SCHAEBS, F.S, DESCHNER, T., KARL S., GAUGG A., RANGE F., Comparing different OT administration methods on OT release and owner-directed behaviours, Canine Science Forum, 2018, p. 81.

11. SPELLMAN, B.A., Perspectives on Psychological Science, 7, No. 1, 2012, p. 58.

12. TEMESI, A., THUROCZY, J., BALOGH, L., MIKLOSI, A., Frontiers in Veterinary Science, 4, 2017, Article Number: UNSP 147, DOI: 10.3389/ fvets.2017.00147.

13. NAGASAWA, M., MITSUI, S., EN, S., OHTANI, N., OHTA, M., SAKUMA, Y., ONAKA, T., MOGI, K., KIKUSUI, T., Science, 348, No. 6232, 2015, p. 333, DOI: 10.1126/science.1261022.

14.RATCLIFFE, V.F., MCCOMB, K., REBY, D., Animal Behaviour, 91, 2014, p. 127.

15.YONG, M.H., RUFFMAN, T., Behaviour, 152, No. 11, 2015. p. 1585.

Manuscript received: 26.09 .2018 